\title{
Multilingual Probabilistic Topic Modeling and its Applications in Web Mining and Search
}

\author{
Marie-Francine Moens \\ KU Leuven \\ Celestijnenlaan 200A \\ Leuven, Belgium \\ marie-francine.moens@cs.kuleuven.be
}

\author{
Ivan Vulić \\ KU Leuven \\ Celestijnenlaan 200A \\ Leuven, Belgium \\ ivan.vulicecs.kuleuven.be
}

\begin{abstract}
Multilingual topic models are a fairly novel group of unsupervised, language-independent and generative machine learning models. This tutorial covers all key aspects of their probabilistic framework and demonstrates how to easily integrate these models into frameworks for cross-lingual and multilingual Web mining and search.
\end{abstract}

\section{TUTORIAL GOALS AND MOTIVATION}

In the current international data-driven society, it becomes increasingly important to quickly and correctly find answers to a user's information need, so as not to lose the desired data in a vast ocean of information. Companies are increasingly interested in mining multilingual and multi-jargon Web data, and in linking Web data. In such a multilingual, multi-idiomatic and multi-domain (or simply $m u l t i *$ ) setting there is a need to access and mine information across multiple different languages and jargons, that is, we desire effective and cheap tools for cross-lingual information retrieval and search, semantic similarity, and for cross-language clustering, classification and linking of content.

Multilingual topic models can be effectively trained on largevolume of non-parallel, comparable multi* data and capture the properties of multi* data directly (e.g., multilingual Wikipedia, news, user-generated content, e-commerce data). They offer an elegant way to represent content across different languages, domains and jargons. State-of-the-art multilingual topic models follow the modeling paradigm established for monolingual settings by probabilistic Latent Semantic Analysis (pLSA) and Latent Dirichlet Allocation (LDA) [1]. A multilingual topic model learns topic distributions over documents (the so-called per-document topic distributions) and word distributions over topics (the so-called per-topic word distributions). The set of language-independent topics serves as a bridge between languages, jargons and other multi* content. Each document, regardless of its actual language, can be presented as a mixture of the language-independent cross-lingual concepts, that is cross-lingual topics (modeled by per-document word distributions), while each topic has its own language-specific representation in each language involved in training (modeled by per-topic word distributions). Due to its generic language-independent nature and the power of inference on unseen documents, these mod-

Permission to make digital or hard copies of part or all of this work for personal or classroom use is granted without fee provided that copies are not made or distributed for profit or commercial advantage, and that copies bear this notice and the full citation on the first page. Copyrights for third-party components of this work must be honored. For all other uses, contact the owner/author(s). Copyright is held by the author/owner(s).

WSDM'14, February 24-28, 2014, New York, New York, USA.

ACM 978-1-4503-2351-2/14/02

http://-enter the whole DOI string from rightsreview form confirmation. els have been used in a variety of cross-lingual (or -domain and -jargon, or simply cross-*) tasks that are of large interest for both academia and industry.

\section{TUTORIAL OVERVIEW}

The tutorial covers recent advances in multilingual topic modeling, with an emphasis on its applications in Web mining and search, and e-commerce. It includes: (1) A high-level overview of the key intuitions and assumptions behind topic modeling in general (starting from monolingual settings) and multi* topic modeling in specific; (2) The methodology and mathematical foundations including training and inference (e.g., variational Dirichlet, Gibbs sampling); and (3) The application of these models in various crosslingual and even cross-modal tasks, with a special focus on crosslingual information retrieval, cross-lingual and cross-jargon Web mining and search, and the evaluation of these tasks.

The tutorial is illustrated with practical cases (e.g., $[2,3])$ of using multilingual probabilistic topic models among which are crosslingual event clustering, cross-lingual document classification, crosslingual semantic word similarity, and translation and transliteration mining. The tutorial explains cross-lingual information retrieval starting from simpler query likelihood models [3] and leading to more elaborate retrieval models which combine cross-lingual topical knowledge with the relevance modeling retrieval framework [4] and use the topical knowledge for query expansion [3]. It goes deeper into linking user interests to the Web e-commerce data such as linking pins to webshops, processing user generated content such as summarizing tweets, and utilizing the topical knowledge in recommendation systems [5]. In a broader framework the tutorial is relevant for searching social media content, user profiling, computational advertising and multimodal data mining.

\section{PARTIAL BIBLIOGRAPHY}

[1] D. M. Blei, A. Y. Ng, and M. I. Jordan. Latent Dirichlet Allocation. Journal of Machine Learning Research, 3:993-1022, 2003.

[2] X. Ni, J.-T. Sun, J. Hu, and Z. Chen. Cross lingual text classification by mining multilingual topics from Wikipedia. In WSDM, pages 375-384, 2011.

[3] I. Vulić, W. De Smet, and M.-F. Moens. Cross-language information retrieval models based on latent topic models trained with document-aligned comparable corpora. Information Retrieval, 16(3):331-368, 2013.

[4] I. Vulić and M.-F. Moens. A unified framework for monolingual and cross-lingual relevance modeling based on probabilistic topic models. In ECIR, pages 98-109, 2013.

[5] S. Zoghbi, I. Vulić, and M.-F. Moens. I pinned it. Where can i buy one like it? Automatically linking Pinterest pins to online Webshops. In DUBMOD, pages 9-12, 2013. 